\title{
Diffuse Reflection Diameter in Simple Polygons*
}

\author{
Gill Barequet \\ Department of Computer Science \\ Technion \\ Haifa, Israel \\ barequet@cs.technion.ac.il \\ Eli Fox-Epstein ${ }^{\dagger}$ \\ Department of Computer Science \\ Brown University \\ Providence, RI \\ ef@cs. brown . edu \\ Diane L. Souvaine ${ }^{\dagger}$ \\ Department of Computer Science \\ Tufts University \\ Medford, MA \\ dls@cs . tufts.edu
}

\author{
Sarah M. Cannon ${ }^{\dagger}$ \\ College of Computing \\ Georgia Institute of Technology \\ Atlanta, GA \\ sarah. cannon@gatech.edu \\ Benjamin Hescott \\ Department of Computer Science \\ Tufts University \\ Medford, MA \\ hescott@cs.tufts.edu \\ Csaba D. Tóth ${ }^{\dagger}$ \\ Department of Mathematics \\ California State University Northridge \\ Los Angeles, CA \\ cdtoth@acm.org
}

Andrew Winslow ${ }^{\dagger}$

Départment d'Informatique

Université Libre de Bruxelles

Brussels, Belgium

awinslow@ulb.ac.be

\begin{abstract}
We prove a conjecture of Aanjaneya, Bishnu, and Pal that the minimum number of diffuse reflections sufficient to illuminate the interior of any simple polygon with $n$ walls from any interior point light source is $\lfloor n / 2\rfloor-1$. Light reflecting diffusely leaves a surface in all directions, rather than at an identical angle as with specular reflections.
\end{abstract}

\section{Introduction}

For a light source placed in a polygonal room with mirror walls, light rays that reach a wall at angle $\theta$, with respect to the normal of the wall's surface, also leave at angle $\theta$. In other words, for these specular reflections the angle of incidence equals the angle of reflection (see Fig. 1).

${ }^{*}$ A preliminary version of this work has been published as: G. Barequet, S. M. Cannon, E. Fox-Epstein, B. Hescott, D. L. Souvaine, C. D. Tóth, A. Winslow, Diffuse reflections in simple polygons, Electronic Notes in Discrete Mathematics 44(5) (2013), 345-350.

${ }^{\dagger}$ Supported in part by National Science Foundation grant CCF-0830734. 


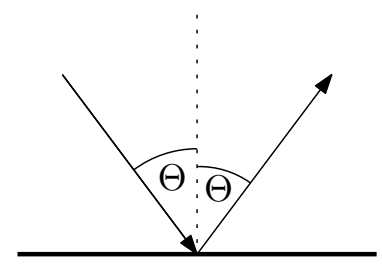

specular

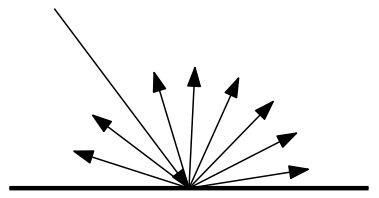

diffuse

Figure 1: Two types of reflections. Specular reflection occurs on mirrored surfaces (left) and diffuse reflection occurs on matte surfaces (right).

Klee [10] asked whether the interior of any room defined by a simple polygon with mirrored walls is completely illuminated by placing a single point light anywhere in the interior. Tokarsky [15] gave a negative answer to this question by constructing simple polygons and pairs of points $(s, t)$ such that there is no path from $s$ to $t$ with specular reflections off the walls of the room.
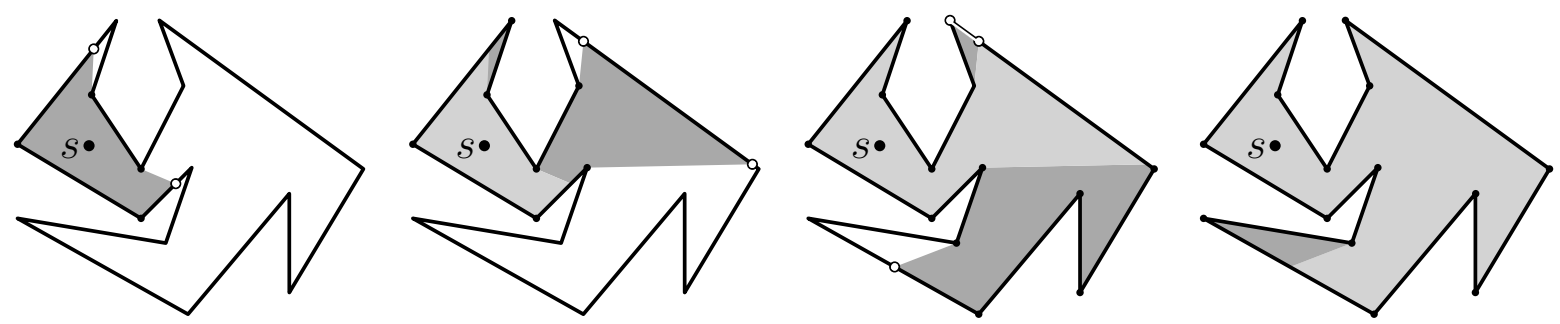

Figure 2: The regions of the polygon illuminated by a light source $s$ after $0,1,2$, and 3 diffuse reflections.

On the other hand, if the walls of the polygonal room $P$ reflect light diffusely in all directions, then it is easy to see that every point in $P$ is illuminated after at most $n$ diffuse reflections (Fig. 2 ). For diffuse reflections, we assume that the vertices of $P$ absorb light, and that light does not propagate along the edges of $P$. A diffuse reflection path is a polygonal path $\gamma$ contained in $P$ such that every interior vertex of $\gamma$ lies in the relative interior of some edge of $P$, and the relative interior of every edge of $\gamma$ is in the interior of $P$.

Aronov et al. 3] were the first to study $V_{k}(s)$, the part of the polygon illuminated by a light source $s$ after at most $k$ diffuse reflections. Formally, $V_{k}(s)$ is the set of points $t \in P$ such that there is a diffuse reflection path from $s$ to $t$ with at most $k$ interior vertices. In particular, $V_{0}(s)$ is the visibility region of point $s$ in the interior of $V$ (where the boundary of $P$ is considered opaque), hence it is a simply-connected region with $O(n)$ edges [6]. Aronov et al.proved that $V_{1}(s)$ is simply connected with at most $\Theta\left(n^{2}\right)$ edges. Brahma et al. 7] constructed simple polygons and a source $s$ such that $V_{2}(s)$ is not simply connected, and showed that $V_{3}(s)$ can have as many as $\Omega(n)$ holes. Extending the work of [3, Aronov et al. [4, 5] and Prasad et al. [12] bounded the complexity of $V_{k}(s)$ at $O\left(n^{9}\right)$ and $\Omega\left(n^{2}\right)$ for all $k$. It remains an open problem to close the gap between these bounds for $k \geq 2$.

Finding a shortest diffuse reflection path between two given points in a simple polygon by brute force is possible in $O\left(n^{10}\right)$ time using the result of Aronov et al. [5]. Ghosh et al. [9] presented a 3 -approximation in $O\left(n^{2}\right)$ time, and their approximation applies even if the polygon $P$ has holes. 

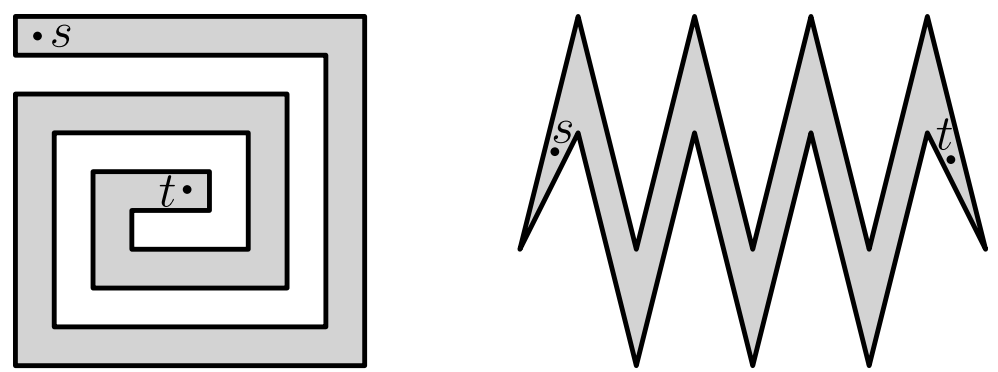

Figure 3: Left: An orthogonal spiral polygon with $n=20$ vertices [1, where every diffuse reflection path between $s$ and $t$ has at least $\lceil n / 2\rceil-2=8$ turns. Right: A zig-zag polygon with $n=16$ vertices where every diffuse reflection path between $s$ and $t$ has at least $\lfloor n / 2\rfloor-1=7$ reflections.

Results We determine the minimum number of diffuse reflections sufficient to illuminate the interior of any simple polygon with $n$ vertices from any interior point $s$. For a simple polygon $P$, the diffuse reflection diameter $D(P)$ is the smallest $k \in \mathbb{N}_{0}$ such that for every two points $s, t \in \operatorname{int}(P)$, there is a diffuse reflection path between $s$ and $t$ with at most $k$ interior vertices (i.e., with at most $k$ reflections). For an integer $n \geq 3$, let $D(n)$ be the largest diffuse reflection diameter $D(P)$ over all simple polygons $P$ with $n$ vertices. Aanjaneya et al. [1] conjectured that $D(n) \leq\lceil n / 2\rceil-1$ and constructed a family of polygons that yields $D(n) \geq\lfloor n / 2\rfloor-2$; see Fig. 3 (left). The family of zig-zag polygons (Fig. 3, right) shows that $D(n) \geq\lfloor n / 2\rfloor-1$ for all $n \geq 3$. Here we prove that this bound is tight.

Theorem 1. We have $D(n)=\lfloor n / 2\rfloor-1$ for every integer $n \geq 3$.

When the points $s$ and $t$ are allowed to be on the boundary of $P$, the minimum number of diffuse reflections may be larger, since a diffuse reflection path cannot have edges along the boundary of $P$. Similarly to $D(P)$, we define $\bar{D}(P)$ as the smallest $k \in \mathbb{N}_{0}$ such that for every two points $s, t \in P$ (in the interior or on the boundary of $P$ ), there is a diffuse reflection path between $s$ and $t$ with at most $k$ interior vertices. For $n \geq 3$, let $\bar{D}(n)$ be the maximum $\bar{D}(P)$ over all simple polygons $P$ with $n$ vertices. We determine $\bar{D}(n)$ for all $n \geq 3$.

Theorem 2. We have $\bar{D}(3)=2$ and $\bar{D}(n)=\lfloor n / 2\rfloor$ for every integer $n \geq 4$.

Related Results for Link Paths The diffuse reflection path is a special case of a link path, which has been studied extensively due to its applications in motion planning, robotics, and curve compression [8, 11]. The link distance between two points, $s$ and $t$, in a simple polygon $P$ is the minimum number of edges in a polygonal path between $s$ and $t$ that lies entirely in $P$. In a polygon $P$ with $n$ vertices, the link distance between two points can be computed in $O(n)$ time [13. The link diameter of $P$, the maximum link distance between any two points in $P$, can be computed in $O(n \log n)$ time [14]. By contrast, no polynomial time algorithm is known for computing the diffuse reflection diameter of a simple polygon.

\section{Preliminary Definitions}

For a set $S \subseteq \mathbb{R}^{2}$, let $\operatorname{int}(S)$ and $\operatorname{cl}(S)$ denote the interior of $S$ and the closure of $S$, respectively. The boundary of $S$, denoted $\partial S$, is $\operatorname{cl}(S) \backslash \operatorname{int}(S)$. The relative interior of a line segment $p q$ in the 
plane is denoted relint $(p q)$. Let $d(p, q)$ be the Euclidean distance between points $p$ and $q$ in the plane.

Let $P$ be a simple closed polygonal domain (for short, simple polygon) with $n$ vertices, where $n \geq 3$. We say that two points $s, t \in P$ see each other (or, are visible to each other) if relint $(s t) \subset$ $\operatorname{int}(P)$. In particular, consecutive vertices of a diffuse reflection path see each other 1

A chord of $P$ is a closed line segment $a b$, such that $a, b \in \partial P$ and $\operatorname{relint}(a b) \subset \operatorname{int}(P)$. Two line segments (e.g., chords of $P$ ) cross each other if there is a point in the relative interior of both segments, but the two segments are not collinear. We define the visibility polygon of a line segment $a b$ of $P$, denoted $V_{0}(a b)$, as the set of points visible from some point in relint $(a b)$. $\left(V_{0}(a b)\right.$ is also known as the weak visibility polygon of the relative interior of $a b$ [6].) A subset $U$ of $P$ weakly covers an edge $e$ of $P$ if $U$ intersects relint $(e)$.

\section{$3 \quad$ A Sequence of Regions $R_{k}$}

Let $P$ be a simple polygon with $n$ vertices, and let $s \in P$. Instead of tackling $V_{k}(s)$ directly, we recursively define an infinite sequence of simply-connected regions $R_{0} \subseteq R_{1} \subseteq R_{2} \subseteq$... such that $R_{0}=V_{0}(s)$ and $R_{k} \subseteq V_{k}(s)$ for all $k \in \mathbb{N}$. In Section 4 , we prove $\operatorname{int}(P) \subseteq R_{\lfloor n / 2\rfloor-1}$ for all $s \in \operatorname{int}(P)$ and $n \geq 3$, which immediately implies Theorem 1. In Section 2 , we prove $P \subseteq R_{\lfloor n / 2\rfloor}$ for all $s \in P$ and $n \geq 4$, which implies Theorem 2 .

Let $R_{0}=V_{0}(s)$. In the remainder of this section, we recall a few well-known characteristics of $V_{0}(s)$, and then formulate properties (i)-(iv) that we wish to maintain for all $R_{k}, k \in \mathbb{N}_{0}$. Using (i)-(iv), we define $R_{k}, k \in \mathbb{N}$, recursively, and show that (i)-(iv) are maintained in each step. Finally, we prove $R_{k}(s) \subseteq V_{k}(s)$ for all $k \in \mathbb{N}_{k}$.
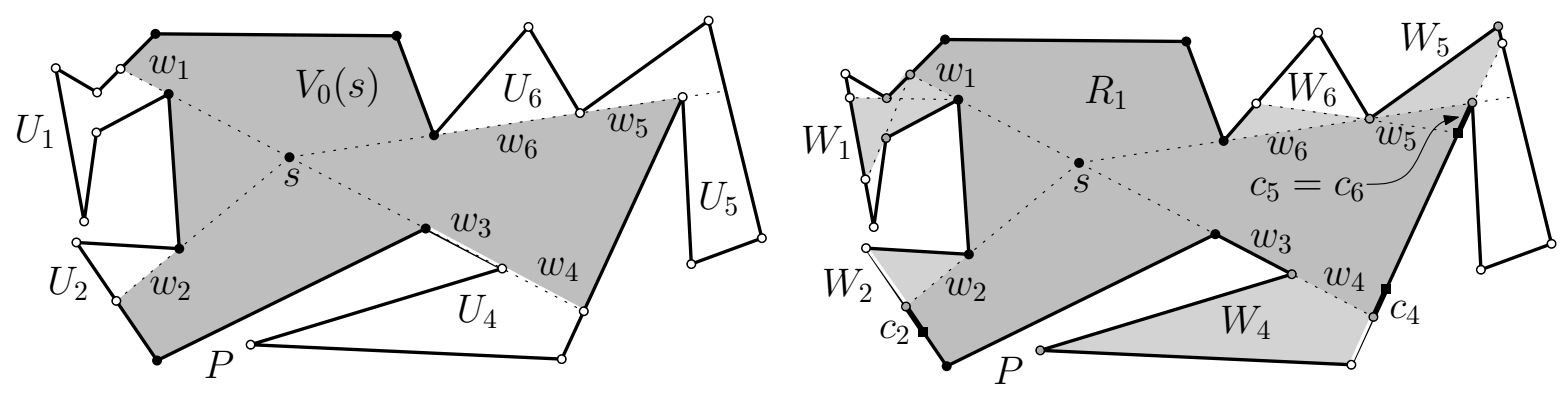

Figure 4: Left: A simple polygon $P$ with a light source $s$. The visibility polygon $V_{0}(s)$ has six windows: $w_{1}, \ldots, w_{6}$. Window $w_{3}$ is the only degenerate window. Windows $w_{1}$ and $w_{3}$ are saturated, while the other windows are unsaturated. Right: $R_{1}$ is the union of the closure of $V_{0}(s)$ and the visibility polygons $W_{w_{i}}$ for $i=1, \ldots, 6$.

Properties of $V_{0}(s) \quad$ Recall that $V_{0}(s)$ is the set of all points $t \in P$ such that $\operatorname{relint}(s t) \subseteq \operatorname{int}(P)$. Refer to Fig. 4. As such, $V_{0}(s)$ is the union of (an infinite number of) closed line segments, each of which is incident to $s$ and some point in $\partial P$, hence $V_{0}(s)$ is simply connected. Consequently, the boundary of $V_{0}(s)$ consists of some line segments along $\partial P$ and possibly segments lying on rays emitted by $s$, which may contain a chord of $P$. However, $V_{0}(s) \cap \partial P$ contains only one point along

\footnotetext{
${ }^{1}$ Note that a more relaxed definition of visibility, that requires only $s t \subset P$, is common in the literature $[$ ]
} 
each ray emitted by $s$. For a set $U$, where $U \subseteq P$, we define a window of $U$ to be a chord of $P$ contained in $\partial U$, or an edge $e$ of $P$ such that $e \subset \partial U$ but relint $(e) \not \subset U$. A window which is an edge of $P$ is called a degenerate window. See Fig. 4 for examples.

If the vertices of $P$ and $s$ are in general position (that is, no three points in a line), then every ray emitted by $s$ contains at most one window $V_{0}(s)$, and no window is degenerate. In general, however, a ray may contain several collinear windows $V_{0}(s)$, some of which may be degenerate (Fig. 4). Suppose that $a b$ is a window of $V_{0}(s)$ such that $a$ lies in the interior of $s b$. For a window $a b$ of $V_{0}(s)$, consider the maximal line segment $a^{\prime} b^{\prime}$ such that $a b \subseteq a^{\prime} b^{\prime}$ and $a^{\prime} b^{\prime} \subseteq \partial V_{0}(s)$ (possibly, $\left.a b=a^{\prime} b^{\prime}\right)$. Then the rays emitted by $s$ can reach $\partial P$ in a neighborhood of $b^{\prime}$, and a sufficiently small neighborhood contains a segment $c \subseteq \partial P$, where relint $(c)$ is visible from the other endpoint $a$ of the window. The windows of $V_{0}(s)$ that lie on distinct rays are necessarily disjoint. Consequently, the region $R_{0}=V_{0}(s)$ satisfies the following properties.

(i) The closure of $R_{k}, \operatorname{cl}\left(R_{k}\right)$, is a simple polygon whose boundary consists of chords of $P$ and line segments contained in the boundary of $P$.

(ii) The endpoints of every window of $R_{k}$ can be labeled by $a$ and $b$ such that $\operatorname{cl}\left(R_{k}\right)$ has an interior angle of at least $180^{\circ}$ at $a$.

(iii) For every window $a b$ of $R_{k}$, there is a nontrivial line segment $c$ such that an endpoint of $c$ lies on the ray $\overrightarrow{a b}$, relint $(c)$ lies in the relative interior of an edge of $P$, relint $(c) \subset R_{k}$, and $a$ sees every point in $\operatorname{relint}(c)$.

(iv) Any two windows of $R_{k}$ are disjoint or collinear; and for any two adjacent windows, there is a common segment $c$ that satisfies property (iii).

Recursive construction of $R_{k}, k \geq 1$ We can now construct $R_{k+1}$ for all $k \in \mathbb{N}_{0}$, assuming that $R_{k}$ is already defined and satisfies (i)-(iv). Intuitively, we construct $R_{k+1}$ by extending $R_{k}$ beyond each of its windows with a visibility region as follows (refer to Fig. 4). A window $a b$ of $R_{k}$ is saturated if every chord of $P$ that crosses $a b$ has an endpoint in $R_{k}$; otherwise, it is unsaturated. Note that every degenerate window is saturated, because a degenerate window crosses no chords.

Each nondegenerate window $a b$ of $R_{k}$ decomposes $P$ into two simple polygons; let $U_{a b}$ denote the polygon that is disjoint from $\operatorname{int}\left(R_{k}\right)$. For a degenerate window, let $U_{a b}=a b$. For each window $a b$, we define a set $W_{a b}$ as follows. If $a b$ is saturated, then let $W_{a b}=V_{0}(a b) \cap U_{a b}$. If $a b$ is unsaturated, then let $c \subset R_{k} \cap \partial P$ be the segment described in property (iii), and let $W_{a b}=V_{0}(c) \cap U_{a b}$. Let $R_{k+1}$ be the union of $\operatorname{cl}\left(R_{k}\right)$ and the sets $W_{a b}$ for all windows $a b$ of $R_{k}$. The definition of the regions $R_{k}, k \in \mathbb{N}_{0}$, readily implies that properties (i)-(iv) are maintained for $R_{k+1}$.

Proposition 1. Let $P$ be a simple polygon and $s \in P$. For every $k \in \mathbb{N}_{0}$, region $R_{k}$ satisfies properties (i)-(iv).

Proof. We proceed by induction on $k \in \mathbb{N}_{0}$. For $k=0$, the region $R_{0}$ is the visibility polygon $V_{0}(s)$ of point $s$ in $P$, and properties (i)-(iv) are easily verified (see Figure 4 , left). Suppose $R_{k}$ satisfies (i)-(iv) for some $k \in \mathbb{N}_{0}$. If $R_{k}$ has no window, then $\operatorname{cl}\left(R_{k}\right)=P$ and $R_{k+1}=P$, hence properties (i)-(iv) trivially hold for $R_{k+1}$. If $R_{k}$ has at least one window, then $R_{k+1}$ is the union of $\operatorname{cl}\left(R_{k}\right)$ and the visibility polygons $W_{a b}$ for all windows $a b$. By definition, $W_{a b}$ contains relint $(a b)$ for both saturated and unsaturated window $a b$. Each $W_{a b}$ satisfies properties (i)-(iv) within $U_{a b}$. This proves properties (i)-(iii) for $R_{k+1}$, and (iv) for windows adjacent in each $W_{a b}$. 
It remains to establish (iv) for pairs of windows, $w$ and $w^{\prime}$, of $R_{k+1}$ that lie on the boundary of $W_{a b}$ and $W_{a^{\prime} b^{\prime}}$, where $a b$ and $a^{\prime} b^{\prime}$ are distinct windows of $R_{k}$. Suppose that their common endpoint is $x=w \cap w^{\prime}$. Then $x$ is also a common endpoint of $a b$ and $a^{\prime} b^{\prime}$. Since $R_{k}$ satisfies (iv) by the induction hypothesis, the windows $a b$ and $a^{\prime} b^{\prime}$ are collinear, and they have a common segment $c$ satisfying (iii). Consequently, $w$ and $w^{\prime}$ lie on the same side of $a b \cup a^{\prime} b^{\prime}$. Note that $a b$ is unsaturated, otherwise $W_{a b}$ would weakly cover the edge of $U_{a b}$ incident to $x$, and $w$ could not be incident to $x$. Analogously, $a^{\prime} b^{\prime}$ is unsaturated. However, if both $a b$ and $a^{\prime} b^{\prime}$ are unsaturated, then $V_{0}(c)$ weakly covers the edge of $U_{a b}$ or $U_{a^{\prime} b^{\prime}}$ incident to $x$. Therefore, at most one of $w$ and $w^{\prime}$ can be incident to $x$. We conclude that the windows $w$ and $w^{\prime}$ of $R_{k+1}$ are disjoint, proving property (iv) for $R_{k+1}$.

The next proposition justifies that the closure of $R_{k}$ is contained in $V_{k+1}(s)$ if $R_{k} \subseteq V_{k}(s)$ and $s \in \operatorname{int}(P)$.

Proposition 2. Let $s \in \operatorname{int}(P)$ and $k \in \mathbb{N}_{0}$. For every set $U \subseteq V_{k}(s)$, we have $\operatorname{cl}(U) \subseteq V_{k+1}(s)$.

Proof. Let $p \in \partial U \backslash U$. Since $p \in \operatorname{cl}(U)$ and $\operatorname{cl}(U) \subseteq \operatorname{cl}\left(V_{k}(s)\right)$, we have $s \in \operatorname{cl}\left(V_{k}(p)\right)$ by symmetry. For every $i \in \mathbb{N}$, there is a point $s_{i} \in V_{k}(p)$ lying in a $\frac{1}{i}$-neighborhood of $s$ such that there is a diffuse reflection path $\left(p, r_{i}(1), \ldots, r_{i}(\ell), s_{i}\right)$ with $\ell \leq k$, where the points $r_{i}(1), \ldots, r_{i}(\ell)$ lie in the interior of some edges of $P$.

By construction, we have $\lim _{i \rightarrow \infty} d\left(s_{i}, s\right)=0$, and we may assume by compactness that there is a point $r \in \partial P$ such that $\lim _{i \rightarrow \infty} d\left(r_{i}(\ell), r\right)=0$. The ray $\overrightarrow{r_{i}(\ell) s_{i}}$ hits $\partial P$ at a point $q_{i}$, and we may assume that there is a point $q \in \partial P$ such that $\lim _{i \rightarrow \infty} d\left(q_{i}(\ell), q\right)=0$, where $s$ lies on the chord $r q$. For a sufficiently large $i \in \mathbb{N}$, there is a point $q^{\prime} \in \partial P$ in a neighborhood of $q$ that lies in the interior of some edge of $P$ and directly sees both $r_{i}(\ell)$ and $s$. Consequently, there is a diffuse reflection path $\left(p, r_{i}(1), \ldots, r_{i}(\ell), q^{\prime}, s\right)$ of length at most $k+1$ between $p$ and $s$. It follows that $p \in V_{k+1}(S)$, and so $\operatorname{cl}(U) \subseteq V_{k+1}(s)$ as desired.

Corollary 1. If $s \in \operatorname{int}(P)$, then $R_{k} \subseteq V_{k}(s)$ for all $k \in \mathbb{N}_{0}$.

Proof. We prove the statement by induction on $k$. In the base case we have $R_{0}=V_{0}(s)$ by definition. Suppose $R_{k} \subseteq V_{k}(s)$ for some $k \in \mathbb{N}_{0}$. By Proposition $2, \operatorname{cl}\left(R_{k}\right) \subseteq V_{k+1}(s)$. For every window $a b$ of $R_{k}$, we show that $W_{a b} \subseteq V_{k+1}$. Specifically, consider the two cases in the construction of $W_{a b}$.

First, suppose $a b$ is a saturated window of $R_{k}$. Then every point $t \in W_{a b}$ sees some point $x \in \operatorname{relint}(a b)$, and $t x$ is contained in a chord ty of $P$, where $y \in R_{k}$. If $y$ is in the relative interior of an edge of $P$, then a diffuse reflection path from $s$ to $y$ can be extended to $t$ using a diffuse reflection at $y$. Otherwise, note that $t$ also sees some neighborhood of $x$ within $\operatorname{relint}(a b)$, hence some neighborhood of $y$ within $\partial P \cap R_{k}$. Again, a diffuse reflection path from $s$ to such a point can be extended to $t$.

Now suppose that $a b$ is unsaturated. Then every point $t \in W_{a b}$ sees a point in the relative interior of segment $c$, where relint $(c) \subseteq R_{k}$ and relint $(c)$ lies in the relative interior of an edge of $P$. A diffuse reflection path from $s$ to any point in $c$ can be extended to $t$ via a diffuse reflection in $c$. In both cases, we have shown $W_{a b} \subset V_{k+1}(s)$. Consequently, $R_{k+1} \subseteq V_{k+1}(s)$.

Weakly covered edges We associate two crucial parameters with the regions $R_{k}, k \in \mathbb{N}_{0}$. For every $k \in \mathbb{N}_{0}$, let $\mu_{k}$ be the number of edges of $P$ weakly covered by $R_{k}$, and $\lambda_{k}$ the total number of windows of $R_{k}$. We derive a lower bound on the number of new edges weakly covered in each round. 
Lemma 1. For every $k \in \mathbb{N}_{0}$,

(1) We have $\mu_{k+1} \geq \mu_{k}+\lambda_{k}$; and

(2) If all windows of $R_{k}$ are saturated, then $\mu_{k+1} \geq \min \left(\mu_{k}+\lambda_{k}+1, n\right)$.

Proof. Recall that $\operatorname{cl}\left(R_{k}\right) \subseteq R_{k+1}$, and so $R_{k+1}$ contains all degenerate windows of $R_{k}$. Now consider nondegenerate windows of $R_{k}$.

Let $a b$ be a nondegenerate window of $R_{k}$. By property (ii), $a$ is a flat or reflex vertex of $\operatorname{cl}\left(R_{k}\right)$, hence it is a convex vertex of $U_{a b}$. Let $a d$ denote the edge of $P$ incident to $a$ and on the boundary of $U_{a b}$. It is clear that $R_{k}$ does not weakly cover $a d$, and we show that $W_{a b}$ weakly covers it. If $a b$ is saturated, it is clear that $W_{a b}$ weakly covers $a d$. If $a b$ is unsaturated, then $U_{a b}$ and $c$ lie on opposite sides of the line spanned by $a b$, and so every point in $c$ sees some part of $a d$ in a neighborhood of $a$. Consequently, $R_{k+1}$ weakly covers at least one new edge of $P$ behind every window of $R_{k}$.

For the second claim, assume that all windows of $R_{k}$ are saturated, but $\mu_{k+1}<n$. Then there is a saturated window $a b$ such that $R_{k+1}$ does not weakly cover all edges of $P$ in $U_{a b}$. As above, let $a d$ denote the edge of $U_{a b}$ incident to $a$, and also let $e$ denote the edge of $P$ that contains $b$ and has nontrivial intersection with the boundary of $U_{a b}$. From above, we know that $R_{k+1}$ weakly covers $a d$. Next, consider all chords of $P$ that cross $a b$ and are parallel to $a d$ or $e$. At least one of these chords has an endpoint in the relative interior of some edge of $P$ that is disjoint from $R_{k}$ and is not $a d$, and so $W_{a b}$ weakly covers at least two new edges of $P$ behind $a b$, as required.

Corollary 2. For every $k \in \mathbb{N}_{0}$,

(1) We have $\mu_{k+1} \geq \min \left(\mu_{k}+1, n\right)$; and

(2) If all windows of $R_{k}$ are saturated, then $\mu_{k+1} \geq \min \left(\mu_{k}+2, n\right)$.

Proof. Note that if $\operatorname{cl}\left(R_{k}\right) \neq P$, then $R_{k}$ has at least one window and $\lambda_{k} \geq 1$.

\section{Counting Weakly Covered Edges in $R_{k}$}

Let $P$ be a simple polygon with $n$ vertices, and let $s \in \operatorname{int}(P)$. In this section, we establish the inequality

$$
\mu_{k} \geq \min (2 k+3, n)
$$

for all $k \in \mathbb{N}_{0}$, which immediately implies Theorem 1. It is folklore that $V_{0}(s)$ weakly covers at least three edges, hence $\mu_{0} \geq 3$.

Proposition 3. If $s \in \operatorname{int}(P)$, then $V_{0}(s)$ weakly covers at least three edges of $P$. Consequently, $\mu_{0} \geq 3$.

Proof. In any triangulation of $P, s$ lies in some triangle whose vertices partition the edges of $\partial P$ into three sets. At least one edge is seen by $s$ in each of the three sets.

We prove $\star \star$ for all $k \in \mathbb{N}_{0}$ by induction on $k$. Recall that $R_{0}$ satisfies $(\star)$ by Proposition 3 , and $\mu_{k}$ strictly monotonically increases until it reaches $n$ by Corollary 2 . Consequently, if $(\star)$ fails for some $R_{k+1}, k \in \mathbb{N}_{0}$, then $R_{k}$ must satisfy ( $\star$ with equality, and $\mu_{k}<n$. This motivates the following definition. A region $R_{k}$ is called critical if $\mu_{k}=2 k+3$ and $\mu_{k}<n$.

By Lemma 1, it is enough to show that whenever $R_{k}$ is critical, then $\lambda_{k} \geq 2$ or all windows of $R_{k}$ are saturated. For every critical region $R_{k}$, we will inductively show (Lemma 2(3)) that one of the following two conditions holds: 
(A) All windows of $R_{k}$ are saturated; or

(B) $R_{k}$ has an unsaturated window and $\lambda_{k} \geq 2$.

Note that these two conditions are mutually exclusive, that is, a region $R_{k}$ cannot satisfy both.

Initialization We first show that $R_{0}$ satisfies one of the two conditions.

Proposition 4. Region $R_{0}$ satisfies condition $(A)$ or $(B)$.

Proof. First, suppose $\lambda_{0} \geq 2$. Then either all windows are saturated so (A) holds, or at least one window is unsaturated and (B) holds.

Next, suppose $\lambda_{0}=1$. If the single window of $R_{0}$ is degenerate, then (A) holds. Assume that $R_{0}$ has exactly one window that is nondegenerate, denoted by $a b$ as defined in (ii). Recall that $R_{0}=V_{0}(s)$, and so every point in $\partial R_{0}$ is contained in a window or directly visible from $s$. Consequently, all points in $\partial R_{0} \backslash a b$ are in $R_{0}$. As $a b$ splits $P$ into $R_{0}$ and $U_{a b}$, every chord of $P$ that crosses $a b$ has exactly one endpoint in $\partial R_{0} \backslash a b$ and so has one endpoint in $R_{0}$, as desired. It follows that window $a b$ is saturated, and so $R_{0}$ satisfies condition (A).

We will also inductively show (Lemma 2) that no two consecutive critical regions satisfy (B). For the first two regions, $R_{0}$ and $R_{1}$, this is established as follows.

Proposition 5. If both $R_{0}$ and $R_{1}$ are critical and $R_{0}$ satisfies (B), then $R_{1}$ satisfies $(A)$.

Proof. If both $R_{0}$ and $R_{1}$ are critical, then $\mu_{0}=3$ and $\mu_{1}=5$. By the proof of Proposition 4, $R_{0}$ must have at least two disjoint windows, otherwise it satisfies (A) instead of (B). Additionally $R_{0}$ must have at most two windows, as otherwise $\lambda_{0} \geq 3$ and so $\mu_{1}>5$ by Lemma 1(1), a contradiction.

Denote by $a_{1} b_{1}$ and $a_{2} b_{2}$ the two disjoint windows of $R_{0}$. By assumption at least one of these two disjoint windows is unsaturated, and it follows that the other must be either unsaturated or saturated and degenerate. The boundary of $R_{0}$ consists of five line segments: window $a_{1} b_{1}$, window $a_{2} b_{2}$, and three segments along three edges of $P$ weakly covered by $R_{0}$.

Since $a_{1} b_{1}$ and $a_{2} b_{2}$ are disjoint and thus not adjacent, they are both incident to some edge $e$ of $P$ weakly covered by $R_{0}$. By Property (ii), we may assume that $a_{1}$ and $a_{2}$ are reflex vertices of $\operatorname{cl}\left(R_{0}\right)$. If $a_{1}$ or $a_{2}$ is incident to $e$, then both windows are saturated, contradicting our earlier observation. Therefore, neither $a_{1}$ nor $a_{2}$ is incident to $e$, hence both $b_{1}$ and $b_{2}$ lie on $e$. Then $R_{1}=\operatorname{cl}\left(R_{0}\right) \cup V_{0}\left(c_{1}\right) \cup V_{0}\left(c_{2}\right)$, where $c_{1}, c_{2} \subseteq e$. Since $R_{1}$ weakly covers precisely one new edge of $P$ behind each window, every window of $R_{1}$ is collinear with $e$. It follows that a chord of $P$ that crosses any window of $R_{1}$ cannot have an endpoint on edge $e$. Therefore one endpoint of such a chord is in $R_{1}=\operatorname{cl}\left(R_{0}\right) \cup V_{0}\left(c_{1}\right) \cup V_{0}\left(c_{2}\right)$, and all windows of $R_{1}$ are saturated. As desired, $R_{1}$ satisfies $(\mathrm{A})$.

The next proposition explores the case where $R_{k+1}$ is critical for some $k \geq 1$, but the previous region $R_{k}$ satisfies $(\star)$ with a strict inequality so is not critical.

Proposition 6. Suppose that $R_{k}$ is not critical and $\mu_{k}>2 k+3$, but $R_{k+1}$ is critical. Then $\lambda_{k}=1$, region $R_{k}$ has an unsaturated window, and $R_{k+1}$ satisfies $(A)$. 

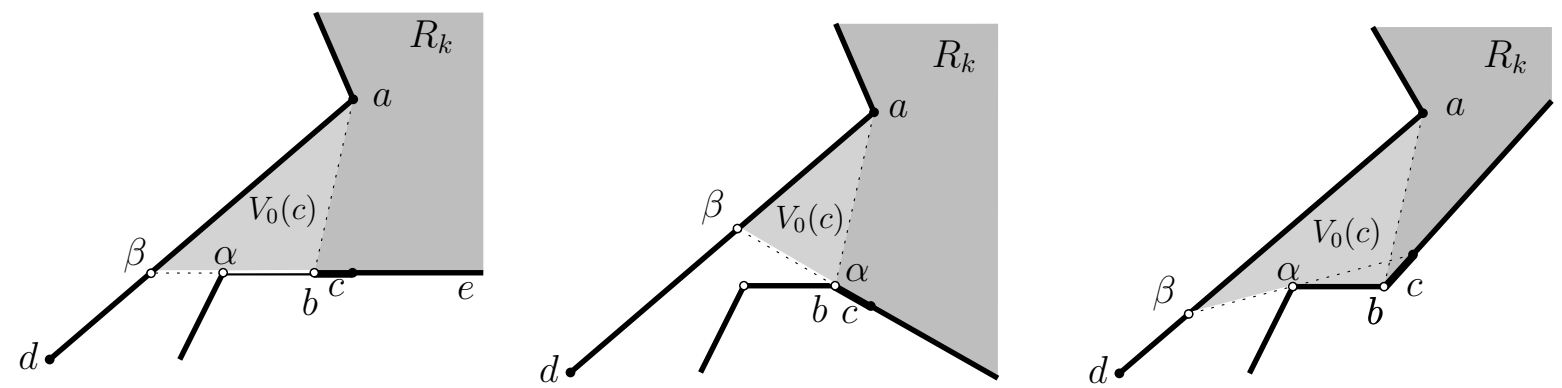

Figure 5: The situation in Proposition 6. The region $R_{k}$ is noncritical and $R_{k+1}$ is critical. The window $a b$ of $R_{k}$ is unsaturated, while the window $\alpha \beta$ of $R_{k+1}$ is saturated. Left: $b$ lies in the relative interior of an edge of $P$. Middle: $b$ is a reflex vertex of $P$. Right: $b$ is a convex vertex of $P$.

Proof. Because $\mu_{k} \geq 2 k+4$ and $\mu_{k+1}=2(k+1)+3=2 k+5$, we have $\mu_{k+1} \leq \mu_{k}+1$. By Corollary 2(1), we have $\mu_{k}=2 k+4$ and $\lambda_{k}=1$, that is, $R_{k}$ has exactly one window.

The window $a b$ cannot be saturated by Corollary 2(2). Since $R_{k+1}$ is critical, we have $\mu_{k+1}<n$ and thus $R_{k+1}$ also has at least one window, which must be within $U_{a b}$. Because $a b$ is unsaturated, $R_{k+1}=\operatorname{cl}\left(R_{k}\right) \cup W_{a b}$, where $W_{a b}=V_{0}(c) \cap U_{a b}$ for a segment $c$ described in (iii). Since $\mu_{k+1}=\mu_{k}+1$, the region $R_{k+1}$ weakly covers precisely one more edge than $R_{k}$. Let $a d$ be the edge of $P$ incident to $a$ lying on the boundary of $U_{a b}$. Refer to Fig. 5. As argued in the proof of Lemma 1, ad is the only edge weakly covered by $W_{a b}$ but not weakly covered by $R_{k}$. We distinguish between two cases to define a point $\beta \in a d$.

Case 1: $b \in \operatorname{relint}(e)$ for some edge $e$ of $P$ (Fig. 5, left). Then $c \subset \operatorname{relint}(e)$. Since $a d$ is the only edge in $U_{a b}$ visible from $c$, the supporting line of $e$ intersects $a d$, and we denote the intersection point by $\beta$.

Case 2: $b$ is a vertex of $P$. In this case, $b$ must be a reflex vertex of $P$ (as in Fig. 5 , middle), otherwise $c$ would also see the edge of $U_{a b}$ incident to $b$ (as in Fig. 5, right). Since $a d$ is the only edge in $U_{a b}$ visible from $c$, the supporting line of $c$ intersects $a d$, and we denote the intersection point by $\beta$.

In both cases, we have $W_{a b}=\Delta(a b \beta) \backslash b \beta$, and so any window of $R_{k+1}$ is contained in $b \beta$. Every chord of $P$ that crosses $b \beta$ has an endpoint in either relint $(a \beta)$ or in $\operatorname{cl}\left(R_{k}\right)$. In either case, one endpoint of such a chord is in $R_{k+1}$, and so all windows of $R_{k+1}$ are saturated.

Induction Step The next three propositions concern the situation where several consecutive regions are critical.

Proposition 7. If all windows of both $R_{k}$ and $R_{k+1}$ are collinear, and $R_{k}$ satisfies $(A)$, then $R_{k+1}$ also satisfies $(A)$.

Proof. Let $\alpha \beta$ be an arbitrary window of $R_{k+1}$. Then $\alpha \beta$ lies on the boundary of some visibility region $W_{a b}$, where $a b$ is a window of $R_{k}$. Since $a b$ is saturated, we have $W_{a b}=V_{0}(a b) \cap U_{a b}$. Consider a chord $c d$ of $P$ that crosses $\alpha \beta$ with $d \in U_{\alpha \beta}$. We need to show that $c \in R_{k+1}$. If $c d$ crosses $a b$, then $c \in R_{k} \subseteq R_{k+1}$ since $a b$ is saturated. If $c=a$ or $c=b$, then $c \in R_{k+1}$ since $a, b \in \operatorname{cl}\left(R_{k}\right) \subseteq R_{k+1}$. Otherwise, $c \in \partial W_{a b} \backslash(a b \cup \alpha \beta)$. Since $a b$ is saturated, all points of $\partial W_{a b} \backslash W_{a b}$ are in windows of $R_{k+1}$, which are collinear with $\alpha \beta$, so $c$ must lie in $W_{a b}$. In all cases, $c \in R_{k+1}$, and so the window $\alpha \beta$ is saturated. 
Proposition 8. If $R_{k}$ and $R_{k+1}$ are critical and $R_{k}$ satisfies (A), then $R_{k+1}$ satisfies (A) or (B).

Proof. First, note that $\mu_{k}=2 k+3$ and $\mu_{k+1}=2 k+5$ by criticality. Note that $R_{k}$ has at least one window, otherwise $\mu_{k+1}=n$, contradicting the criticality of $R_{k+1}$. If $\lambda_{k} \geq 2$, then by Lemma $1(2)$, $\mu_{k+1} \geq(2 k+3)+2+1>2 k+5$, which is a contradiction. It follows that $R_{k}$ has exactly one window that is nondegenerate.

If $\lambda_{k+1} \geq 2$, then $R_{k+1}$ satisfies (A) or (B): either all windows are saturated and (A) holds, or it has an unsaturated window and (B) holds. If $\lambda_{k+1} \leq 1$, then any window of $R_{k+1}$ is saturated by Proposition 7 and so (A) holds.

Proposition 9. Suppose that $R_{k}, R_{k+1}$, and $R_{k+2}$ are critical, $R_{k}$ satisfies (A), and $R_{k+1}$ satisfies (B). Then $R_{k+2}$ satisfies (A).

Proof. Note that $\mu_{k}=2 k+3, \mu_{k+1}=\mu_{k}+2$, and $\mu_{k+2}=\mu_{k+1}+2$. By Lemma 1 $(2), R_{k}$ has only one window, which is saturated; label this window $a b$ as described in Property (ii). Recall that in this case, $W_{a b}=V_{0}(a b) \cap U_{a b}$. We have $\lambda_{k+1}=2$, as condition (B) yields $\lambda_{k+1} \geq 2$ and Lemma 1(1) implies $\lambda_{k+1} \leq 2$. However, $R_{k+1}$ cannot have two adjacent collinear windows, otherwise both of these windows would be saturated by Proposition 7, and $R_{k+1}$ would satisfy (A). By property (iv), $R_{k+1}$ has exactly two windows that are disjoint. We denote them $\alpha_{1} \beta_{1}$ and $\alpha_{2} \beta_{2}$, respectively. Refer to Fig. 6.

By Property (ii), we may assume that $a$ is a reflex vertex or a straight vertex of $\operatorname{cl}\left(R_{k}\right)$. Denote by $a d$ the edge of $P$ on the boundary of $U_{a b}$ and incident to $a$. Since $\mu_{k+1}=\mu_{k}+2$, region $R_{k+1}$ weakly covers precisely two new edges of $P$ : one is ad and call the other $e$. Let $f$ be the edge of $P$ containing $b$ and weakly covered by $U_{a b}$. Note that $\alpha_{1} \beta_{1}$ and $\alpha_{2} \beta_{2}$ are disjoint and lie on the boundary of $W_{a b}$. Since $a d$ and $f$ can each be incident to at most one of them, we have $e \neq f$, and edge $e$ is incident to both $\alpha_{1} \beta_{1}$ and $\alpha_{2} \beta_{2}$.

The boundary of $W_{a b}$ is formed by segments $a b, \alpha_{1} \beta_{1}$, and $\alpha_{2} \beta_{2}$, and some part of the edges $a d, e$, and possibly $f$. Note that $a d \subset \partial W_{a b}$ since a point in $a b$ sufficiently close to $a$ can see all of $a d$. However, the intersection $f \cap \partial W_{a b}$ could be the single point $b$, or a nontrivial line segment connecting $b$ and an endpoint of $f$. Without loss of generality, we may assume that $\partial W_{a b}$ contains, in counterclockwise order, $\alpha_{1} \beta_{1}$, part of $e, \alpha_{2} \beta_{2}, a d, a b$, and possibly part of $f$, but contains no other segments.

Since $\mu_{k+2}=\mu_{k+1}+2$, region $R_{k+2}$ weakly covers precisely one new edge of $P$ behind each of the two windows $\alpha_{1} \beta_{1}$ and $\alpha_{2} \beta_{2}$. It follows that $R_{k+2}$ has at most two windows: at most one behind each of $\alpha_{1} \beta_{1}$ and $\alpha_{1} \beta_{2}$.

By Property (ii) and disjointness of $W_{a b}$ 's two windows, we may assume that $\alpha_{1}$ and $\alpha_{2}$ are reflex vertices of $\operatorname{cl}\left(R_{k+1}\right)$. Since $W_{a b}=V_{0}(a b) \cap U_{a b}$, the region $W_{a b}$ has a reflex or flat interior angle at both $\alpha_{1}$ and $\alpha_{2}$. We have $\alpha_{1} \in f$ (possibly $\alpha_{1}=b$ ), and $\alpha_{2}=d$. Consequently, both $\beta_{1}$ and $\beta_{2}$ are contained in $e$. A segment in $P$ can connect two points in $\operatorname{relint}\left(\alpha_{1} \beta_{1}\right)$ and $\operatorname{relint}\left(\alpha_{2} \beta_{2}\right)$, respectively. Therefore, $\alpha_{1} \beta_{1}$ and $\alpha_{2} \beta_{2}$ are unsaturated or degenerate windows.

Let $\gamma_{1}$ and $\gamma_{2}$, respectively, be the segments $c$ described in (iii) for the windows $\alpha_{1} \beta_{1}$ and $\alpha_{2} \beta_{2}$. Note that both $\gamma_{1}$ and $\gamma_{2}$ are in relint $(e)$. By construction, $R_{k+2}=\operatorname{cl}\left(R_{k+1}\right) \cup V_{0}\left(\gamma_{1}\right) \cup V_{0}\left(\gamma_{2}\right)$. Since $R_{k+2}$ weakly covers exactly one new edge of $P$ behind each of $\alpha_{1} \beta_{1}$ and $\alpha_{2} \beta_{2}$, every window of $R_{k+2}$ is collinear with $e$. It follows that a chord of $P$ that crosses any window of $R_{k+2}$ cannot have an endpoint on the edge $e$, which contains the only uncovered portions of $\partial R_{k+2}$ that are on the boundary of $P$. Therefore one endpoint of such a chord is in $R_{k+2}=\operatorname{cl}\left(R_{k+1}\right) \cup V_{0}\left(\gamma_{1}\right) \cup V_{0}\left(\gamma_{2}\right)$. Consequently, any window of $R_{k+2}$ is saturated. 

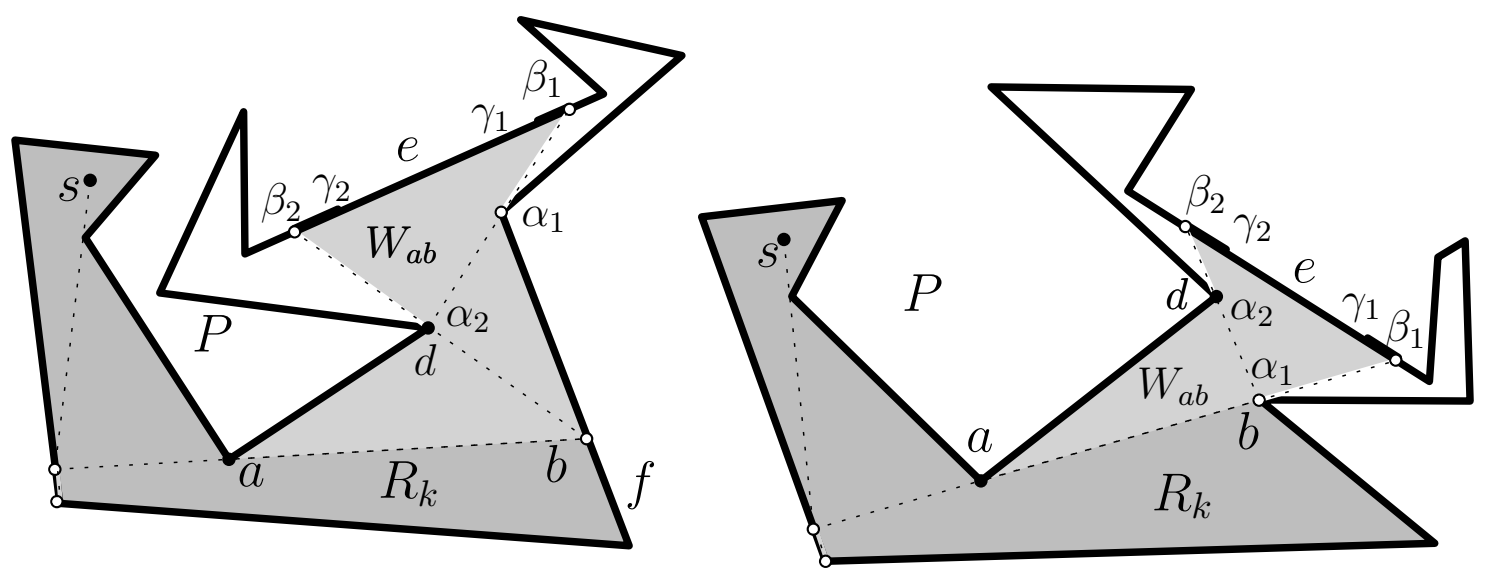

Figure 6: The situation in Proposition 9, The region $R_{k}$ has a unique window $a b$, and $W_{a b}$ weakly covers two new edges of $P$ : $a d$ and $e . R_{k+1}$ has two windows $\alpha_{1} \beta_{1}$ and $\alpha_{2} \beta_{2}$. Left: $b$ lies in the relative interior of an edge $f$ of $P$. Right: $b$ is a vertex of $P$ and $b=\alpha_{1}$.

We are now in position to prove Lemma2. We note that claim (1) of the lemma is the statement we want to prove, and we are able to do this by establishing a stronger induction argument also maintaining claims (2) and (3).

Lemma 2. For all $k \in \mathbb{N}_{0}$,

(1) $\mu_{k} \geq \min (2 k+3, n)$;

(2) If $R_{k}$ is critical, it satisfies (A) or (B); and

(3) If $R_{k}$ is critical and satisfies (B), then either $R_{k-1}$ is critical and satisfies (A), or $k=0$.

Proof. First, suppose $k=0$. Then $\mu_{0} \geq 3$ by Proposition 3, satisfying (1). By Proposition $4, R_{0}$ satisfies (A) or (B), proving (2). Claim (3) trivially holds for $k=0$.

For the inductive step, suppose $k \geq 1$ and that (1), (2), and (3) hold for all smaller $k$. First, we establish (1). If $R_{k-1}$ is critical, then by the induction hypothesis it must satisfy (A) or (B). By criticality, we have $\mu_{k-1}=2 k+1$, and Lemma 1 yields $\mu_{k} \geq \min \left(\mu_{k}+2, n\right)=\min (2 k+3, n)$. If $R_{k-1}$ is not critical, then $\mu_{k-1} \geq \min (2 k+1, n)$ by the induction hypothesis and $\mu_{k-1} \neq 2 k+1$ by the definition of criticality. Consequently $\mu_{k-1} \geq \min (2 k+2, n)$, and Corollary 2(1) yields $\mu_{k} \geq \mu_{k-1}+1 \geq \min (2 k+3, n)$, proving (1).

To establish (2) and (3), suppose $R_{k}$ is critical. If $R_{k-1}$ is not critical, then $\mu_{k-1} \geq 2 k+2$ from the discussion above. Applying Proposition 6 (for $k-1$ instead of $k$ ), it follows that $R_{k}$ satisfies (A). If $R_{k-1}$ is critical and satisfies (A), then $R_{k}$ satisfies (A) or (B) by Proposition 8. It remains to consider the case that $R_{k-1}$ is critical and satisfies (B).

Suppose that both $R_{k}$ and $R_{k-1}$ are critical and $R_{k-1}$ satisfies (B). Claim (3) implies (for $k-1$ instead of $k$ ) that either $k=1$ or $R_{k-2}$ is critical and satisfies (A). If $k=1$, then $R_{k}$ satisfies (A) by Proposition 5. If $R_{k-2}$ is critical and satisfies (A), we apply Proposition 9 (for $k-2$ instead of $k$ ) and conclude that $R_{k}$ satisfies (A). In all cases, $R_{k}$ satisfies (A) or (B), proving (2). If $R_{k}$ satisfies (B), then $R_{k-1}$ satisfies (A), proving (3).

We can now finally prove Theorem 1 . 
Theorem 1. We have $D(n)=\lfloor n / 2\rfloor-1$ for every integer $n \geq 3$.

Proof. We prove that in every simple polygon $P$ with $n \geq 3$ vertices, there exists a diffuse reflection path with at most $\lfloor n / 2\rfloor-1$ reflections between any two points $s, t \in \operatorname{int}(P)$. It is enough to show that $\operatorname{int}(P) \subseteq V_{k}(s)$ for every $s \in \operatorname{int}(P)$ and every $k \geq\lfloor n / 2\rfloor-1$.

Note that $\lfloor n / 2\rfloor-1=\lceil(n-3) / 2\rceil$. By Lemma 2 , $\mu_{\lfloor n / 2\rfloor-1} \geq 2(\lfloor n / 2\rfloor-1)+3 \geq n$, so $R_{\lfloor n / 2\rfloor-1}$ weakly covers all edges of $P$. It follows that $R_{\lfloor n / 2\rfloor-1}$ does not have any window, otherwise Lemma 1 would imply that $P$ has an edge that is not weakly covered. Therefore $\operatorname{int}(P) \subseteq R_{\lfloor n / 2\rfloor-1}$, as claimed. Corollary 1 then implies $\operatorname{int}(P) \subseteq V_{\lfloor n / 2\rfloor-1}(s)$, proving the theorem.

\section{Diffuse Reflection Paths between Boundary Points}

In this section, we prove Theorem 2. If the light source $s$ is in the interior of the polygon $P$, then Theorem 1 and Proposition 2 readily imply $P \subseteq R_{\lfloor n / 2\rfloor} \subseteq V_{\lfloor n / 2\rfloor}(s)$. It remains to consider diffuse reflection paths between points $s, t \in \partial P$, that is, between points on the boundary of $P$. If $s$ is a vertex of $P$, then no other points on the edges of $P$ incident to $s$ are illuminated by $s$. For example, in a triangle $P$, a diffuse reflection path between two vertices requires two turns (Fig. 7, left), and consequently $\bar{D}(P)=2$.
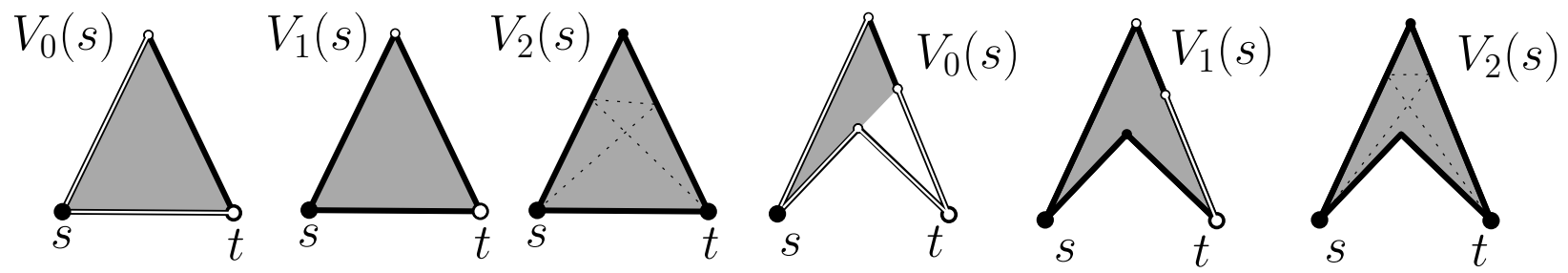

Figure 7: The regions of a triangle (resp., a nonconvex quadrilateral) illuminated by the light source $s$ at the vertex after 0,1 , and 2 diffuse reflections.

Let $s \in \partial P$, where $P$ is a simple polygon. We cannot use Proposition 2 when $s \in \partial P$. Proposition 2 is replaced by the following weaker statement.

Proposition 10. Let $s \in \partial P$ and $k \in \mathbb{N}_{0}$. Suppose that $U \subseteq V_{k}(s)$ such that $\operatorname{cl}(U)$ is a simple polygon. Then, $V_{k+1}(s)$ contains all points of $\partial U$ with the possible exception of the convex vertices of $\operatorname{cl}(U)$; and we have $\operatorname{cl}(U) \subseteq V_{k+2}(s)$.

Proof. Let $p \in \partial U \backslash U$. For every $i \in \mathbb{N}$, there is a point $p_{i} \in V_{k}(s)$ lying in a $\frac{1}{i}$-neighborhood of $p$ such that there is a diffuse reflection path $\left(s, r_{i}(1), \ldots, r_{i}(\ell), p_{i}\right)$ with $\ell \leq k$, where the points $r_{i}(1), \ldots, r_{i}(\ell)$ lie in the interior of some edges of $P$. By perturbing $p_{i}$, if necessary, we may assume that the ray $\overrightarrow{r_{i}(\ell) p_{i}}$ hits the boundary of $P$ at a point $q_{i}$ lying in the relative interior of an edge of $P$. If $i \in \mathbb{N}$ is sufficiently large, then $q_{i}$ directly sees $p$, unless $p$ and $q_{i}$ lie on the same edge of $P$, which means that $p$ is a convex vertex of $\operatorname{cl}(U)$. Consequently, if $p$ is not a convex vertex of $\operatorname{cl}(U)$, then $\left(s, r_{i}(1), \ldots, r_{i}(\ell), q_{i}, p\right)$ is a diffuse reflection path of length at most $k+1$ from $s$ to $p$. If $p$ is a convex vertex of $\operatorname{cl}(U)$, then there is a point $r_{i}$ in the visibility polygon of $q_{i}$ that directly sees $p$, and so $\left(s, r_{i}(1), \ldots, r_{i}(\ell), q_{i}, r_{i}, p\right)$ is a diffuse reflection path of length at most $k+2$ from $s$ to $p$. 
We can define $R_{k}$ analogously to Section 3 . Let $R_{0}=V_{0}(s)$; and for $k \geq 1$, let $R_{k}$ be the union of $R_{k-1}$, the sets $W_{a b}$ for all windows $a b$ of $R_{k-1}$, the boundary $\partial R_{k-1}$ with the exception of the convex vertices of $\operatorname{cl}\left(R_{k-1}\right)$, and $\operatorname{cl}\left(R_{k-2}\right)$ if $k \geq 2$. Proposition 1 holds for $R_{k}$ for all $k \in \mathbb{N}_{0}$; and similarly to Corollary 1 , we have $R_{k} \subseteq V_{k}(s)$ for all $k \in \mathbb{N}$.

Recall that $\mu_{k}$ is the number of edges of $P$ weakly covered by region $R_{k}$, and $\lambda_{k}$ is the number of windows of $R_{k}$. Instead of ( $夫$ ), we maintain the following inequality for all $k \in \mathbb{N}$ :

$$
\mu_{k} \geq \min (2 k+2, n)
$$

Inequality $\star \star$ combined with Proposition 11 below, readily implies Theorem 2.

Proposition 11. If $k \geq 1$ and $R_{k}$ weakly covers all edges of $P$ (i.e., $\left.\mu_{k}=n\right)$, then $V_{k+1}(s)=P$.

Proof. Since $\mu_{k}=n$, then $R_{k}$ has no windows and int $(P) \subseteq R_{k}$. By Proposition 10, $R_{k+1}$ contains $\partial P$ with the possible exception of the convex vertices of $P$. As $R_{k} \subseteq R_{k+1}$ and $R_{k+1} \subseteq V_{k+1}(s)$, it only remains to show that all convex vertices of $P$ are in $V_{k+1}(s)$. Consider a convex vertex $v$ of $P$. If $v \in \partial R_{k-1}$, then $v \in R_{k+1}$ by Proposition 10. Suppose $v \in \partial R_{k}$ but $v \notin \partial R_{k-1}$. Then $v$ is incident to some region $U_{a b}$ separated from $R_{k-1}$ by a window $a b$, where $\operatorname{int}\left(U_{a b}\right) \subseteq W_{a b}$. If $U_{a b}$ is saturated, then all boundary points of $U_{a b}$ are in $R_{k}$, hence in $R_{k+1}$ and $V_{k+1}(s)$. If $U_{a b}$ is unsaturated, then $\operatorname{int}\left(U_{a b}\right)$ is visible from a segment $c \in R_{k-1}$ described in property (iii). Since $v \notin R_{k}$, vertex $v$ is incident to the edge of $P$ that contains $c$. In this case, however, there is a diffuse reflection path from $c$ to $v$ with one reflection, and as $c \in R_{k-1} \subseteq V_{k-1}(s)$, then $v \in V_{k+1}(s)$, as desired.

We argue that $\star \star \star$ holds for all $k \in \mathbb{N}$. Lemma 1 holds when $s \in \partial P$, but some of the propositions in Section 4 require adjustments. Proposition 3 (i.e., $\mu_{0} \geq 3$ ) is replaced by the following:

Proposition 12. If $s \in \partial P$, then $R_{0}=V_{0}(s)$ weakly covers at least one edge of $P$, and $R_{1}$ weakly covers at least $\min (n, 4)$ edges of $P$.

Proof. As argued in the proof of Proposition 3 , the boundary of $R_{0}=V_{0}(s)$ contains line segments from at least three edges of $P$. Hence $\operatorname{cl}\left(R_{0}\right)$ weakly covers at least three edges of $P$. However, $s$ cannot see any point in the edges of $P$ that contain $s$. At most two edges of $P$ contain $s$, hence $V_{0}(s)$ weakly covers at least one edge of $P$.

All interior points of the edges of $\partial R_{0}$ can be reached from $s$ after one diffuse reflection. Hence the region $R_{1}$ covers at least three edges of $P$ that are weakly covered by $\operatorname{cl}\left(R_{0}\right)$. This completes the proof for $n=3$. If $n \geq 4$, then either $\operatorname{cl}\left(R_{0}\right)=P$ and so $R_{1}$ weakly covers all edges of $P$, or $R_{0}$ has a window and $R_{1}$ covers at least one edge behind the window by Lemma $1(1)$.

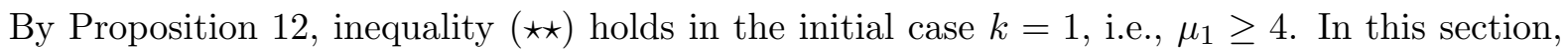
we consider a region $R_{k}$ critical if $\mu_{k}=2 k+2$ and $\mu_{k}<n$. Conditions (A) and (B) can now be adapted verbatim. Propositions 4 and 5 are replaced by a single claim about $R_{1}$ :

Proposition 13. If $R_{1}$ is critical, then $R_{1}$ satisfies (A).

Proof. If $R_{1}$ is critical, then $\operatorname{cl}\left(R_{1}\right) \neq P$, and so $R_{0}$ and $R_{1}$ each have at least one window. Recall that $\operatorname{cl}\left(R_{0}\right)$ weakly covers at least three edges of $P$, and $R_{1}$ weakly covers at least one additional edge of $P$ that is not weakly covered by $\operatorname{cl}\left(R_{0}\right)$. Since $R_{1}$ is critical, we have $\mu_{1}=4$, hence $\operatorname{cl}\left(R_{0}\right)$ 
weakly covers precisely three edges of $P$, and $R_{1}$ weakly covers precisely one additional edge $a d$. By Lemma 1, $R_{0}$ has a unique unsaturated window, say $a b$. Refer to Fig. 8.

Because the window of $R_{0}$ is unsaturated, $R_{1}=\operatorname{cl}\left(R_{0}\right) \cup W_{a b}$, where $W_{a b}=V_{0}(c) \cap U_{a b}$ for a segment $c$ described in property (iii) lying in the relative interior of some edge $e$ of $P$. Since the region $R_{1}$ weakly covers only one new edge not weakly covered by $\operatorname{cl}\left(R_{0}\right)$, the supporting line of $e$ intersects $a d$ at some point $\beta$. Denote by $\alpha$ the endpoint of $e$ that lies in the segment $b \beta$. Observe that $W_{a b}=\Delta(a b \beta) \backslash b \beta$, and all windows of $R_{1}$ are contained in $\alpha \beta$, which is collinear with $e$. Every chord of $P$ that crosses $\alpha \beta$ has an endpoint in either the relative interior of $a \beta$ or in $\operatorname{cl}\left(R_{0}\right) \backslash\{e\}$, which are contained in $R_{1}$. As desired, all windows of $R_{1}$ are saturated and $R_{1}$ satisfies (A).

After replacing ( $\star$ with $(\star \star)$ and using the new definition of critical regions, Propositions 6, 6, 8, and 9, as well as Lemma 2, go through, showing that $\star \star \star)$ is maintained for all $k \in \mathbb{N}$. We are now ready to prove Theorem 2 .
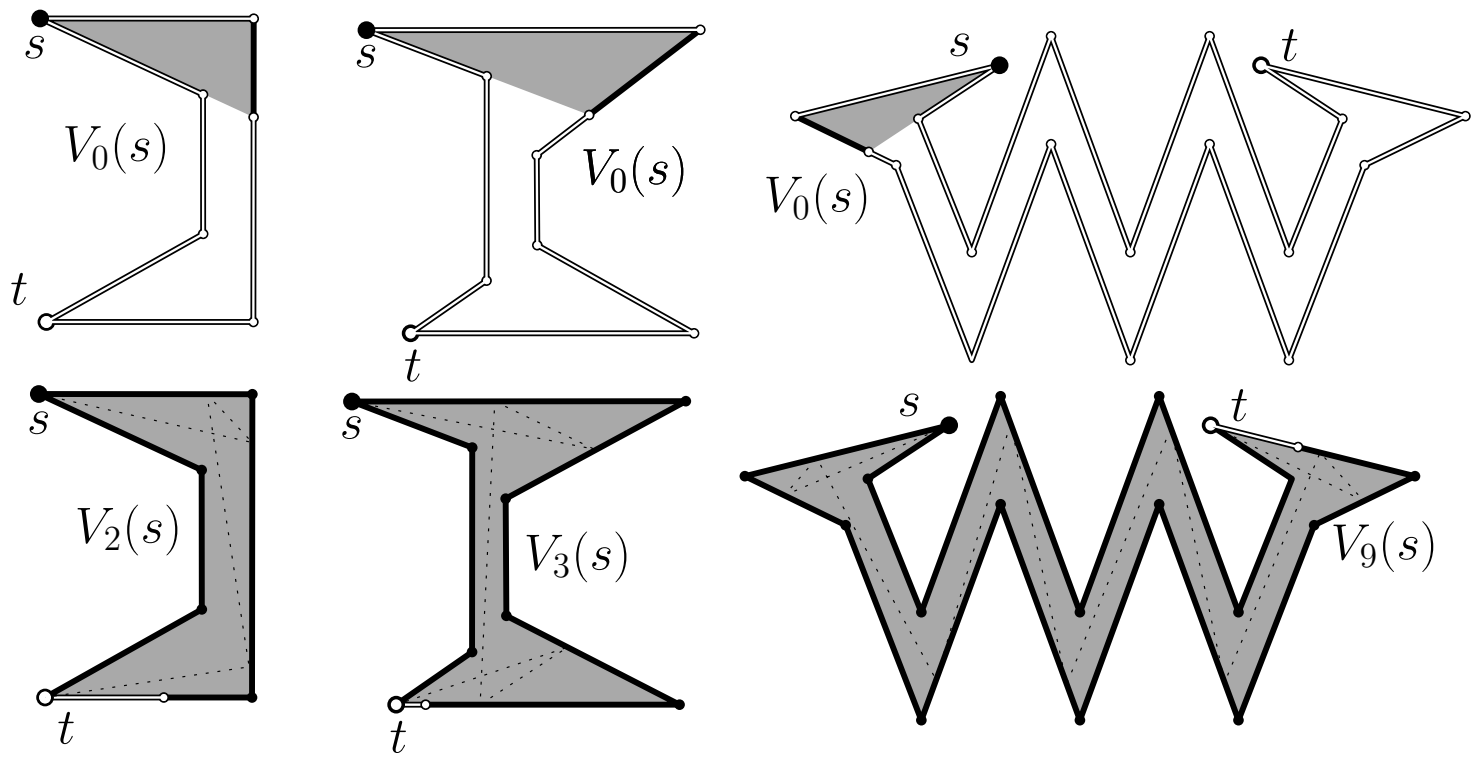

Figure 8: Simple polygons with $n=6,8$, and 18 vertices, where every diffuse reflection path between $s$ and $t$ has at least $\lfloor n / 2\rfloor$ turns. Top row: the regions $V_{0}(s)$. Bottom row: the regions $V_{\lfloor n / 2\rfloor-1}(s)$, which contain the interior $\operatorname{int}(P)$, and all points on the boundary $\partial P$ except for a line segment incident to $t$.

Theorem 2, We have $\bar{D}(3)=2$ and $\bar{D}(n)=\lfloor n / 2\rfloor$ for every integer $n \geq 4$.

Proof. It is easily verified that $D(3)=2$ (see Fig. 7). We show that in every simple polygon $P$ with $n \geq 4$ vertices, there exists a diffuse reflection path with at most $\lfloor n / 2\rfloor$ reflections between any two points $s, t \in P$. Theorem 1 implies that it is enough to prove $P \subseteq V_{k}(s)$ for every $s \in \partial P$ and $k \geq\lfloor n / 2\rfloor$. By $(\star \star), R_{\lceil n / 2\rceil-1}$ weakly covers all edges of $P$. It follows that region $R_{\lceil n / 2\rceil-1}$ does not have any window, and so $\operatorname{int}(P) \subseteq R_{\lceil n / 2\rceil-1}$.

Suppose first that $n$ is even. Then $\operatorname{int}(P) \subseteq R_{\lceil n / 2\rceil-1}$ combined with Proposition 11 yields $P=V_{\lceil n / 2\rceil}(s)$, hence $P=V_{\lfloor n / 2\rfloor}(s)$, as required. Suppose now that $n$ is odd and $n=2 \ell+1$ for some $\ell>1$. By $\star \star \star), R_{\ell-1}$ weakly covers at least $2 \ell=n-1$ edges of $P$. If $R_{\ell-1}$ weakly covers all 
edges of $P$, then $P=V_{\ell}(s)=V_{\lfloor n / 2\rfloor}(s)$ by Proposition 11. Otherwise $R_{\ell-1}$ weakly covers exactly $n-1$ edges of $P$. Consequently, $R_{\ell-1}$ is critical, and we have $\lambda_{\ell-1}=1$ by Lemma 1(1). By Lemma 2(2), $R_{\ell-1}$ has a saturated window $a b$. In this case, either $U_{a b}=a b$ or $U_{a b}$ is a triangle adjacent to $a b$. Hence $U_{a b} \subseteq V_{0}(a b)$, and so $P=R_{\ell}=R_{\lfloor n / 2\rfloor} \subseteq V_{\lfloor n / 2\rfloor}(s)$, as claimed.

The matching lower bound $\bar{D}(n) \geq\lfloor n / 2\rfloor$ for $n \geq 4$ follows from a family construction. For every $n \geq 4$, there is a simple polygon $P_{n}$ with $n$ vertices, including $s, t \in P_{n}$, such that every diffuse reflection path between $s$ and $t$ has $\lfloor n / 2\rfloor$ reflections. For odd $n, n \geq 5$, the polygon $P_{n}$ is obtained by subdividing an arbitrary edge of $P_{n-1}$. The polygon $P_{4}$ is a nonconvex quadrilateral, where $s$ and $t$ are two opposite convex corners (Fig. 7). Polygon $P_{6}$ is depicted in Fig. 8, For even integers $n \geq 8$, the polygon $P_{n}$ is constructed by attaching two nonconvex quadrilaterals to a zigzag polygon as in Fig. 8 .

\section{Conclusion}

We have shown that in every simple polygon with $n$ vertices, every point light source $s \in \operatorname{int}(P)$ can illuminate the interior of $P$ after at most $\lfloor n / 2\rfloor-1$ diffuse reflections, and this bound is the best possible. A point light source $s \in P$, either in the interior or boundary of $P$, can illuminate $P$ after at most $\lfloor n / 2\rfloor$ diffuse reflections for any $n \geq 4$, and this bound is tight. However, the diffuse reflection diameter may be significantly smaller for a given polygon $P$ (e.g., convex polygons). Several problems related to diffuse reflection paths remain open:

- Is there an efficient algorithm for finding the diffuse reflection diameter of a given simple polygon $P$ with $n$ vertices? Combining our result with the bound $O\left(n^{9}\right)$ on the complexity of $V_{k}(s)$ by Aronov et al. [5], we can compute in polynomial time the minimum $k \in \mathbb{N}_{0}$ such that $P=V_{k}(s)$ for any point $s \in P$. But it is unclear how many points $s \in P$ would have to be tested to find the maximum.

- Is there an efficient data structure for a simple polygon $P$ that, for a query point pair $s, t \in$ $\operatorname{int}(P)$, would report a diffuse reflection path between $s$ and $t$ with the minimum number of reflections? Arkin et al. [2] designed a data structure for analogous queries for minimum link paths in a simple polygon.

- What is the maximum diffuse reflection diameter of a star-shaped polygon $S$ with $n$ vertices? Even though every point of $S$ is visible from some point $s \in S$, it is not clear how a diffuse reflection path could take advantage of this property. Our lower bound constructions do not extend to star-shaped polygons.

- What is the maximum diffuse reflection diameter of a simple polygon with $n$ vertices, $r$ of which are reflex? It is clear that no reflection is necessary for $r=0$, but the dependence on the parameter $r$ is not clear.

- What is the maximum diffuse reflection diameter of a polygon with $h$ holes and a total of $n$ vertices?

\section{Acknowledgements}

We would like to thank all reviewers for helpful comments improving the presentation of the paper

and the MIT-Tufts Research Group on Computational Geometry for initial discussions of the problem. 


\section{References}

[1] M. Aanjaneya, A. Bishnu, S. P. Pal, Directly visible pairs and illumination by reflections in orthogonal polygons, in: Proceedings of 24th European Workshop on Computational Geometry, 2008, pp. 241-244.

[2] E. Arkin, J. Mitchell, S. Suri, Logarithmic-time link path queries in a simple polygon, Internat. J. Comput. Geom. Appl. 5 (4) (1995) 75-79.

[3] B. Aronov, A. Davis, T. K. Dey, S. P. Pal, D. C. Prasad, Visibility with one reflection, Discrete \& Computational Geometry 19 (4) (1998) 553-574.

[4] B. Aronov, A. Davis, T. K. Dey, S. P. Pal, D. C. Prasad, Visibility with multiple reflections, Discrete \& Computational Geometry 20 (1) (1998) 61-78.

[5] B. Aronov, A. Davis, J. Iacono, A. S. C. Yu, The complexity of diffuse reflections in a simple polygon, in: J. R. Correa, A. Hevia, M. Kiwi (Eds.), LATIN 2006: Theoretical Informatics, Vol. 3887 of LNCS, Springer Berlin Heidelberg, 2006, pp. 93-104.

[6] T. Asano, S. K. Ghosh, T. Shermer, Visibility in the plane, in Handbook of Computational Geometry (J.-R. Sack and J. Urrutia, eds.), North-Holland, Amsterdam, 2000, pp. 829-876.

[7] S. Brahma, S. P. Pal, D. Sarkar, A linear worst-case lower bound on the number of holes inside regions visible due to multiple diffuse reflections, Journal of Geometry 81 (1-2) (2004) 5-14.

[8] S. K. Ghosh, Visibility algorithms in the plane, Cambridge Univ. Press, 2007, Ch. 7, pp. $218-254$.

[9] S. K. Ghosh, P. P. Goswami, A. Maheshwari, S. C. Nandy, S. P. Pal, S. Sarvattomananda, Algorithms for computing diffuse reflection paths in polygons, The Visual Computer 28 (12) (2012) 1229-1237.

[10] V. Klee, Is every polygon illuminable from some point?, American Mathematical Monthly 76 (1969) 180.

[11] A. Maheshwari, J.-R. Sack, H. N. Djidjev, Link distance problems, in: Handbook of Computational Geometry, Elsevier, 2000, Ch. 12, pp. 519-558.

[12] D. C. Prasad, S. P. Pal, T. K. Dey, Visibility with multiple diffuse reflections, Computational Geometry 10 (3) (1998) 187-196.

[13] S. Suri, A linear time algorithm for minimum link paths inside a simple polygon, Comput. Vision. Graph. Image Process. 35 (1986) 99-110.

[14] S. Suri, On some link distance problems in a simple polygon, IEEE Trans. Robot. Autom. 6 (1990) 108-113.

[15] G. Tokarsky, Polygonal rooms not illuminable from every point, The American Mathematical Monthly 102 (10) (1995) 867-879. 